\title{
Independent modal variable structure fuzzy active vibration control of cylindrical thin shells laminated with photostrictive actuators
}

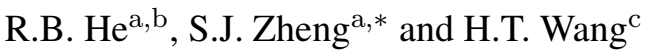 \\ ${ }^{a}$ State Key Laboratory of Mechanics and Control of Mechanical Structures, Nanjing University of Aeronautics and \\ Astronautic, Nanjing, Jiangsu, China \\ ${ }^{\mathrm{b}}$ School of Electrical Information, Anhui University of Technology, Maanshan, Anhui, China \\ ${ }^{c}$ School of Mechanical and Electrical Engineering, Nanjing University of Aeronautics and Astronautics, Nanjing, \\ Jiangsu, China
}

Received 2 July 2012

Revised 1 January 2013

Accepted 22 January 2013

\begin{abstract}
Photostrictive actuator, which can produce photodeformation strains under the activation of ultraviolet lights, is a new promising non-contact photoactuation technique for active vibration control of flexible structures. Generally, the membrane control action plays a major role in vibration control of flexible thin shell structures. However, it is unfortunate that the existing photostrictive actuator configuration can not induce negative membrane control forces. In this paper, a novel multi-layer actuator configuration is first presented to remedy this deficiency, followed by presenting the photostrictive/shell coupling equations of thin cylindrical shells laminated with the proposed multi-layer actuator configuration. Moreover, considering the time-variant and nonlinear dynamic characteristics of photostrictive actuator, variable structure self-adjusting parameter fuzzy active controller is explored to overcome disadvantages of conventional control schemes, in which off-line fuzzy control table is adopted. The optimal switching surface is derived to increase the range of sliding mode to facilitate vibration suppression. A continuous function is used to replace the sign function for reducing the variable structure control chattering. Finally, two case studies are carried out to evaluate the effectiveness of the proposed actuator configuration and the control scheme. Numerical simulation results demonstrate that the proposed actuator configuration is effective in shell actuation and control. It is also suggested that the proposed control strategy could give better control responses than the proportional velocity feedback.
\end{abstract}

Keywords: Photostrictive actuator, multi-layer actuator configuration, cylindrical thin shells, variable structure, fuzzy active vibration control

\section{Introduction}

Over the last decades, smart structures with distributed sensors and actuators designed by various smart materials, such as piezoelectric, electrostrictive, and magnetostrictive actuators, etc. have been extensively studied and evaluated [1-3]. However, these smart material distributed sensors/actuators systems are hard-wired. In many operating environments, hard-wire connections are likely to attract electrical noises that will contaminate the sensor and control signals and degenerate the distributed control. To remedy this situation, optically driven and remotely actuated

\footnotetext{
${ }^{*}$ Corresponding author: S.J. Zheng, State Key Laboratory of Mechanics and Control of Mechanical Structures, Nanjing University of Aeronautics and Astronautic, Nanjing 210016, Jiangsu, China. E-mail: sjzheng@nuaa.edu.cn.
} 
photostrictive actuators have been proposed in place of the conventional electromechanical actuators [4-11]. The photostrictive type of actuator can produce actuation strain as a result of irradiation from high-intensity light. In principle, the photostrictive effect arises from a superposition of the photovoltaic effect and converse piezoelectric effect. When the photostrictive materials are illuminated by high-energy light, a high voltage up to the order of $\mathrm{kV}$ $\mathrm{mm}^{-1}$ is generated along spontaneous polarization. This high photo-voltage then induces mechanical strain due to the converse piezoelectric effect of the photostrictive materials. The photostrictive actuator system has neither electric lead wires nor electric circuits, so it is relatively immune to electromagnetic interferences. Photostrictive actuators can be driven only by the irradiation of light, hence suitable to use as actuators in which lead wires can hardly be connected because of their ultra-small size or of their working conditions such as ultra-high vacuum or outer space.

Among photostrictive materials, PLZT ceramic made from lead $(\mathrm{Pb})$, lanthanum $(\mathrm{La})$, zirconium $(\mathrm{Zr})$ and titanium (Ti) has been researched widely because of its easy fabrication and relatively high photostriction. Fukuda et al. [4, 5] tested the actuating behavior of a bimorph PLZT element irradiated on both sides of the bimorph to increase the response speed to the light irradiation. They also designed and tested an optical servo system using bimorph PLZT. Uchino [6,7] developed a photo-driven relay and a micro-walking device using a bimorph configuration, which had neither electric lead wires nor electric circuits. Poosanaas [8] introduced a solar tracking device for future space missions. Furthermore, PLZT actuators can be used for precision actuation and remote structural control of elastic structures. Constitutive models, which account for the coupling of photostriction, photodeformation, pyroelectricity, thermoelasticity and opto-piezothermoelasticity of one- and two- dimensional distributed PLZT actuators were investigated by Liu and Tzou [9], Shih and Tzou [10], and Tzou and Chou [11]. The PLZT-actuated beams and plates with constant light intensity control method have been recently investigated (Liu and Tzou [9], Morikawa and Nakada [12], Shih and Tzou [13], Shih et al. [14,15], Lei et al. [16]). Shih and Tzou [17] first introduced PLZT actuators to photonic control of flexible cylindrical thin shell structures and focused on considering the effects of actuator location as well as membrane and bending components on the control action. Shih and Tzou [18] and Shih et al. [19] also evaluated the membrane and bending control actions of PLZT-laminated parabolic and spherical shells. Wang et al. [20] investigated two types of control algorithms (i.e., the proportional velocity feedback light intensity control and the constant light intensity control), and evaluated two light illumination schemes (i.e., the uniform illumination and the alternate illumination). Yue et al. [21] explored a new multiple degree-of-freedom (multi-DOF) distributed actuator configuration spatially bonded on the surface of shell structures to enhance the spatial modal controllability. Zheng [22] carried out the finite element simulation of wireless structural vibration control with photostrictive actuators. In the state-of-art studies [17-20], paired PLZT actuators are respectively placed on the top and bottom surfaces of the shell to induce both positive and negative modal bending control moment by alternately activating the light sources. However, there is a misconception that the lower and upper actuators can respectively induce positive and negative membrane control force in the aforementioned studies [17-20]. Wang's experimental investigation [23] demonstrated that the deformation of single piece of PLZT actuator is always positive, which is independent of the light illumination direction. Indeed, a positive modal control force can be induced by the lower actuator when the cylindrical shell oscillates downward, and a negative modal bending control moment can also be induced by the upper actuator when the cylindrical shell oscillates upward, but, unfortunately, the membrane control force is still positive. It is noteworthy that the membrane control action is more significant than the bending control action in cylindrical shells $[17,20,23,24]$. On basis of the combination of membrane force and bending control actions, it is infeasible to induce both positive and negative control forces using the existing photostrictive actuator configuration. This deficiency could even lead to the failure of system control. To remedy this deficiency, it is promised to present a new actuator configuration. In this paper, a novel multi-layer actuator configuration is first explored to induce both positive and negative membrane force.

Then, it is noticed that the characteristic of the active vibration control using photostrictive actuators is different from using piezoelectric actuators. Usually, in active vibration control for the PLZT-actuated flexible structures, the light intensity can only be positive and the deformation of the PLZT actuators is always tensile. Thus, to induce positive and negative modal control force, the light illumination should be alternatively applied to the top and bottom PLZT actuators. In view of the switching actuation, variable structure control (VSC) action takes place. VSC is basically a control law that switches rapidly between two values or gains, with an objective of bringing the system trajectory onto a predetermined switching surface, called the sliding surface. The main features of VSC 
technique are invariance to parameter variation of the system and rejection of external disturbances. However, a major drawback of VSC in practical applications is the chattering problem. Several techniques have been proposed to alleviate this problem [26,27]. In order to implement VSC effectively, the switching surface and control law must be well-designed. From the literatures $[9,12-15,17,20]$, it is found that the velocity surface is chosen as the switching surface, but it is not optimal for active vibration suppression. In this paper, an optimal switching surface is derived. Moreover, as far as the control law is concerned, most of past work $[9,12-15,17,20]$ have focused on the application of two control algorithms (i.e., the proportional velocity feedback control and the constant light intensity control, also called Lyapunov control). According to the literatures $[9,20]$, the effect of the proportional velocity feedback control is not very ideal for the complex nonlinear and time-variant behaviors of the photostrictive actuator. By comparison, the constant light intensity control provides better control responses. But, since the constant control is discontinuous, the feedback may result in unstable responses at high control gains. Recently, fuzzy control has emerged as one of the most active and fruitful approaches to dealing with complex and ill-defined dynamic systems [28-31]. The motivation is often due to the fact that the system knowledge and dynamic behavior are qualitative and uncertain, so the fuzzy set theory appears to provide a suitable representation of such knowledge. Usually, the qualitative knowledge is first represented by fuzzy sets, then, by using the compositional rule of inference and approximate reasoning, a fuzzy control law can be constructed. Compared to conventional control theories, fuzzy logic control does not rely on the analysis of a mathematical model of the plant. In essence, fuzzy logic can emulate human thinking and organize the approximate and indeterminate natures of the environment. Though this method has not been viewed as a rigorous theory, it is believed that the basic idea behind the approach would have a significant influence on the practice of controlling complex systems vibration in the future.

This paper presents an investigation into variable structure fuzzy active vibration control of a simply supported cylindrical thin shell laminated with the novel multi-layer actuator configuration. The novel multi-layer actuator configuration is first explored to induce both positive and negative membrane forces. Based on the modal analysis techniques, modal control equations of the simply supported cylindrical thin shell laminated with the proposed actuator configuration are established. Considering the complex nonlinear and time-variant behaviors being exist in PLZT actuator, variable structure self-adjusting parameters fuzzy active controller is explored to overcome disadvantages of the conventional control schemes. The optimal switching surface is derived to increase the range of sliding area to facilitate vibration suppression. To reduce the variable structure control chattering, the continuous function is used to replace sign function for smoothing out the control discontinuity in a thin boundary layer. In case studies, the effectiveness of the proposed actuator configuration is studied and evaluated firstly, followed by variable structure fuzzy light intensity control and velocity feedback light intensity control. Based on two control algorithms, dynamic responses of transverse displacement, control force, control light intensity, and photo-induced electric field are presented respectively. Numerical simulation results show that the proposed control strategy could effectively suppress vibration.

The remainder of this paper is organized as follows: Section 2 proposes a novel multi-layer actuator configuration and establishes the independent mode vibration control equations of photoelectric laminated cylindrical shell. The proposed variable structure fuzzy active control algorithm is presented in Section 3. Two cases are studied, and several simulation results are shown in Section 4, and Section 5 concludes this paper.

\section{Modeling of photoelectric laminated cylindrical shell based on the proposed novel multi-layer actuators configuration}

\subsection{The proposed new multi-layer actuator configuration}

The 0-1 (along the length or width direction) polarized PLZT actuators have been investigated by a number of researchers $[4-8,11]$. A $0-1$ polarized photostrictive actuator configuration used in bending vibration control of flexible structure usually can be a bulk or a bimorph, as shown in Fig. 1. Though having the same mechanism of photostrictive effect, they have different characteristics and applications. A bulk photostrictive actuator is made of a photostrictive plate, and its two small side surfaces are metallized as two electrodes, while its two largest surfaces are used to receive light input. A bimorph actuator is made of two bulk photostrictive actuators bonded back-to-back 


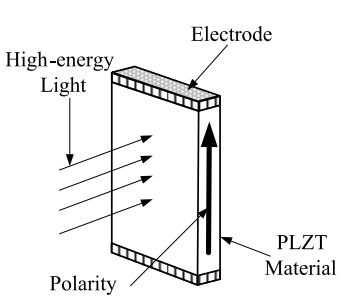

(a)

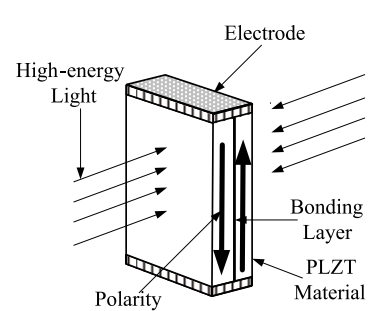

(b)

Fig. 1. Two types of 0-1 polarized photostrictive actuators: (a) A bulk actuator, (b) A bimorph actuator.

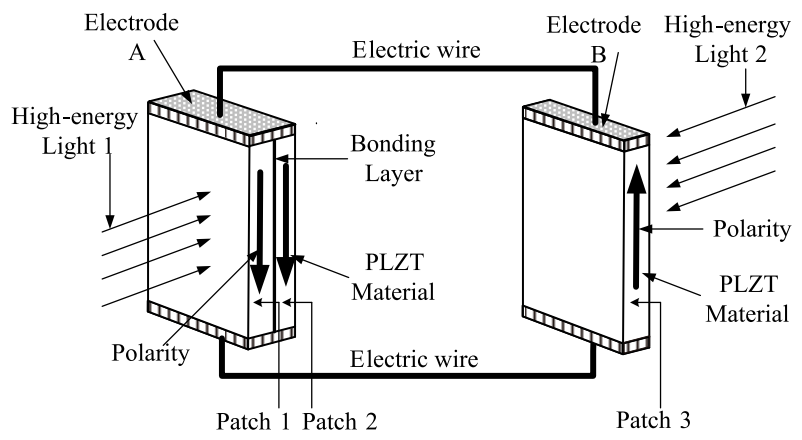

Fig. 2. New multi-layer photostrictive actuator configuration.

using opaque glue. These two actuators are polarized in opposite direction (neglecting the bonding layer viscoelastic role) and the two end surfaces are metallized as common electrodes. When a high-energy light irradiates on one layer of photostrictive actuator, the light can cause this layer to deform in extension and induce an electric field on the irradiated layer. The induced electric field is applied to another layer through the conducting material. The electric field then triggers the converse piezoelectric effect and induces this layer to deform in contraction. Thus, bending motion of the bimorph actuator takes place [4,5,12].

Note that the above-mentioned two configurations can generate positive and negative bending moments as well as a positive membrane control action, but can not generate a negative membrane force. Meanwhile, it is worth stressing that the membrane control action is the dominating for the lower shell modes vibration control [17,20,23,24]. Hence, it is difficult for the existing actuator configurations to achieve vibration suppression for curved shell structures. On the basis of these two configurations, a new actuator configuration is proposed, which is made up of three layers of the same size actuator patch, as shown in Fig. 2. The patches 1 and 2 in the proposed configuration, having identical polarization direction, are bonded back-to-back using opaque glue. Their end surfaces are metalized as two common electrodes. The remaining patch 3 is arranged in opposite polarization direction, and then its upper electrode B is connected with the upper common electrode A of patches 1 and 2 using electric wire, and the bottom electrodes are also connected in the same way. When the high-energy illumination 1 irradiates on patch 1 of the proposed actuator configuration, the light can induce an extensional strain and an electric field on patch 1, which will go through the conducting material and be applied on patches 2 and 3. The voltage then triggers the converse piezoelectric effect in patches 2 and 3. Since the photo-induced electric field is the same as the polarization direction of patch 2 and opposite to that of patch 3, an expansive strain is induced in patch 2, and a compressive strain in patch 3. Likewise, when the high-energy illumination 2 irradiates on patch 3 , the light can induce an extensional strain and an electric field on patch 3, which will be applied on patches 1 and 2 through the conducting material. Since the photo-induced electric field is opposite to the polarization direction of patches 1 and 2, a compressive strain in patches 1 and 2 . As mentioned above, the characteristics of this new actuator configuration are qualitatively analyzed. It is assumed that the actuator patches are laminated perfectly on the upper and bottom surfaces of a simply supported cylindrical shell, as shown in Fig. 3. In next section, a mathematical model of a simply supported cylindrical shell laminated with the proposed actuator configuration will be formulated.

\subsection{Modeling of cylindrical shell laminated with circumferential photostrictive actuators}

A simply supported cylindrical shell laminated with distributed optical actuators is shown in Fig. 4. The mathematical model of cylindrical shell laminated with photostrictive actuators is presented in this section. A cylindrical coordinate system $\left(x, \psi, \alpha_{3}\right)$ is employed, where $x, \psi$, and $\alpha_{3}$ represent the longitudinal, circumferential, and transverse directions, respectively. The actuator location is defined by the coordinates $\left(x_{1}, \psi_{1}\right)$ and $\left(x_{2}, \psi_{2}\right)$, as shown in Fig. 4, which is the same as that of the literature [20]. 


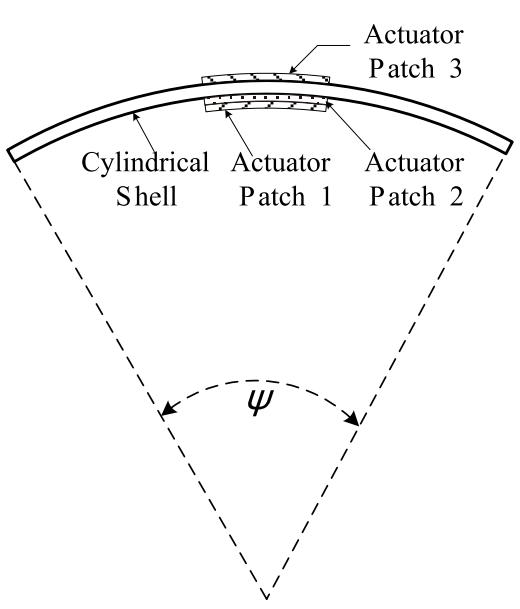

Fig. 3. Profile of a simply supported cylindrical shell laminated with the proposed actuator configuration.

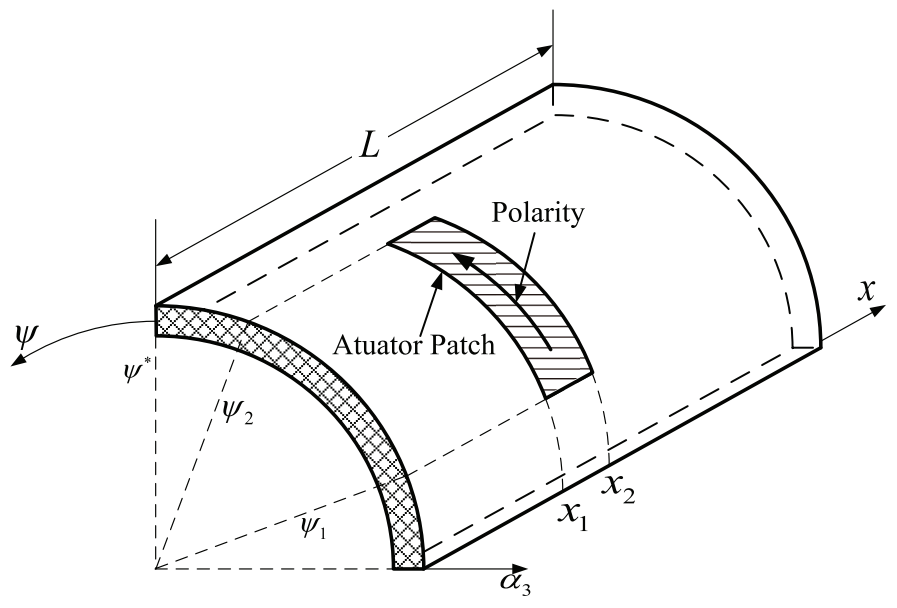

Fig. 4. Cylindrical shell laminated with photostrictive actuators.

For a simply supported cylindrical shell, the transverse dynamic response can be represented by a sum of all participating modal responses [17,20]:

$$
u_{3}(x, \psi, t)=\sum_{m=1}^{\infty} \sum_{n=1}^{\infty} \eta_{i m n}(t) \sin (m \pi x / L) \sin \left(n \pi \psi / \psi^{*}\right)
$$

where $\eta_{i m n}$ is the modal displacement, $m$ is the half-wave number in the $x$ direction, $n$ is the half-wave number in the $\psi$ direction, $L$ and $\psi^{*}$ are the length and curvature angle of the cylindrical shell, respectively.

Based on the governing equilibrium equation of cylindrical shell, the equation of motion control of the cylindrical shell laminated with photostrictive actuators in the transverse direction is formulated as $[9,17,20]$ :

$$
-\frac{\partial^{2} M_{x x}}{\partial x^{2}}-\frac{2}{R} \frac{\partial^{2} M_{x \psi}}{\partial x \partial \psi}-\frac{1}{R^{2}} \frac{\partial^{2} M_{\psi \psi}}{\partial \psi^{2}}+\frac{N_{\psi \psi}}{R}+\rho h \ddot{u}_{3}+c \dot{u}_{3}=\frac{\partial^{2} M_{x x}^{a}}{\partial x^{2}}+\frac{1}{R^{2}} \frac{\partial^{2} M_{\psi \psi}^{a}}{\partial \psi^{2}}-\frac{N_{\psi \psi}^{a}}{R}
$$

where $R$ and $\rho$ are the radius and mass density of the cylindrical shell, respectively; $h$ is the thickness of the cylindrical shell; $N_{i j}$ and $M_{i j}$ denote the elastic forces and moments, respectively; $c$ is the damping constant; $N_{i j}^{a}$ and $M_{i j}^{a}$ represent the membrane control force and bending moment induced by the actuator, respectively.

Substituting the modal expansion Eq. (1) into Eq. (2), integrating over the whole shell surface, and applying the modal orthogonality, the $m n$th modal equation of cylindrical shell can be formulated as $[17,20]$

$$
\ddot{\eta}_{m n}+2 \zeta_{m n} \omega_{m n} \dot{\eta}_{m n}+\omega_{m n}^{2} \eta_{m n}=F_{m n}^{c}
$$

where $\zeta_{m n}$ is the $m n$th modal damping ratio, $\omega_{m n}$ is the modal natural frequency of the $m n$th mode, and $F_{m n}^{c}$ denotes the mnth modal control force.

Because the photostrictive actuator is oriented in the $\psi$ direction, it only induces the control action in the circumferential direction. The circumferential modal control force $F_{m n}^{c}$ can be defined as follows:

$$
F_{m n}^{c}=\frac{4}{\rho h R L \psi^{*}} \int_{0}^{L} \int_{0}^{\psi^{*}}\left(\frac{1}{R^{2}} \frac{\partial^{2} M_{\psi \psi}^{a}}{\partial \psi^{2}}-\frac{N_{\psi \psi}^{a}}{R}\right) \times \sin (m \pi x / L) \sin \left(n \pi \psi / \psi^{*}\right) R \mathrm{~d} x \mathrm{~d} \psi
$$

The membrane force $N_{\psi \psi}^{a}$ and bending moment $M_{\psi \psi}^{a}$ by the actuator are

$$
N_{\psi \psi}^{a}=h_{a} Y_{a} \bar{S}\left[u_{s}\left(x-x_{1}\right)-u_{s}\left(x-x_{2}\right)\right]\left[u_{s}\left(\psi-\psi_{1}\right)-u_{s}\left(\psi-\psi_{2}\right)\right]
$$




$$
M_{\psi \psi}^{a}=\frac{h+h_{a}}{2} h_{a} Y_{a} \bar{S}\left[u_{s}\left(x-x_{1}\right)-u_{s}\left(x-x_{2}\right)\right]\left[u_{s}\left(\psi-\psi_{1}\right)-u_{s}\left(\psi-\psi_{2}\right)\right]
$$

where $u_{s}(\cdot)$ is a step function, $Y_{a}$ is the Young's modulus of the photostrictive actuator, $h_{a}$ is the actuator thickness, and $\bar{S}$ is the photodeformation strain of the photostrictive actuator.

Substituting Eq. (5) into the first and second integral terms of Eq. (4), each term can be rewritten as:

$$
\begin{aligned}
& \frac{4}{\rho h R L \psi^{*}} \int_{0}^{L} \int_{0}^{\psi^{*}} \frac{1}{R^{2}} \frac{\partial^{2} M_{\psi \psi}^{a}}{\partial \psi^{2}} \times \sin (m \pi x / L) \sin \left(n \pi \psi / \psi^{*}\right) R \mathrm{~d} x \mathrm{~d} \psi=-\tilde{M}_{\psi m n} \bar{S} \\
& \frac{4}{\rho h R L \psi^{*}} \int_{0}^{L} \int_{0}^{\psi^{*}}\left(-\frac{N_{\psi \psi}^{a}}{R}\right) \times \sin (m \pi x / L) \sin \left(n \pi \psi / \psi^{*}\right) R \mathrm{~d} x \mathrm{~d} \psi=-\tilde{N}_{\psi m n} \bar{S}
\end{aligned}
$$

where

$$
\tilde{M}_{\psi m n}=\frac{2\left(h+h_{a}\right) h_{a} Y_{a}}{\rho h L R^{2} \psi^{*}} \frac{n L}{m \psi^{*}}\left[\left(\cos \frac{m \pi x_{2}}{L}-\cos \frac{m \pi x_{1}}{L}\right) \times\left(\cos \frac{n \pi \psi_{2}}{\psi^{*}}-\cos \frac{n \pi \psi_{1}}{\psi^{*}}\right)\right]
$$

and

$$
\tilde{N}_{\psi m n}=\frac{4 h_{a} Y_{a}}{\rho h L R \psi^{*}} \frac{\psi^{*} L}{m n \pi^{2}}\left[\left(\cos \frac{m \pi x_{2}}{L}-\cos \frac{m \pi x_{1}}{L}\right) \times\left(\cos \frac{n \pi \psi_{2}}{\psi^{*}}-\cos \frac{n \pi \psi_{1}}{\psi^{*}}\right)\right]
$$

Here, $\tilde{M}_{\psi m n}$ and $\tilde{N}_{\psi m n}$ are used to simplify the expressions. Employing those symbols, the circumferential modal control force $F_{m n}^{c}$ becomes

$$
F_{m n}^{c}=-\left(\tilde{M}_{\psi m n}+\tilde{N}_{\psi m n}\right) \bar{S}
$$

As discussed early, the photodeformation effect induced by the photostrictive actuator involves two stages, i.e., the photovoltaic effect and the converse piezoelectric effect, is defined by $[9,15]$ :

$$
\bar{S}(t)=d_{33}\left[E_{l}(t)+\frac{P_{n}}{\varepsilon} \theta(t)\right]-\frac{\lambda \theta(t)}{Y_{a}}
$$

where $d_{33}$ is the piezoelectric-strain constant, $\lambda$ is a thermal stress coefficient, and $Y_{a}$ is the Young's modulus of the photostrictive actuator, $P_{n}$ is the pyroelectric constant and $\varepsilon$ is the permittivity, and the photo-induced electric field $E_{l}(t)$ and the temperature $\theta(t)$ can be estimated as:

$$
\begin{aligned}
& E_{l}\left(t_{j}\right)=E_{l}\left(t_{j-1}\right)+\left[E_{s}-E_{l}\left(t_{j-1}\right)\right] \frac{\alpha}{a_{s}} I\left(t_{j}\right) e^{-\left(\alpha / a_{s}\right) I\left(t_{j}\right) \Delta t} \Delta t-E_{l}\left(t_{j-1}\right) \beta e^{-\beta \Delta t} \Delta t \\
& \theta\left(t_{j}\right)=\theta\left(t_{j-1}\right)+\left\{\left[I\left(t_{j}\right) P-\gamma \theta\left(t_{j-1}\right)\right] \Delta t\right\} /(H+\gamma \Delta t)
\end{aligned}
$$

where $\Delta t$ is the time step, $E_{s}$ is the saturated photovoltaic field, $\alpha$ is the photostrictive actuator constant, $\beta$ is the voltage leakage constant, $P$ is the power of the absorbed heat, $\gamma$ is the heat transfer rate, $H$ is the heat capacity of the photostrictive actuator, $I\left(t_{j}\right)$ is the light intensity at time $t_{j}$, and $\alpha_{s}=a / b$ (length/width) is the aspect ratio.

As previously mentioned, a high-energy light should be alternatively applied to the top and bottom photostrictive actuators depending on the switching mode, such as the velocity direction. In Fig. 5(a), when the cylindrical shell oscillates downward, the light should be applied to the bottom actuators, and then a high voltage between two electrodes is generated duo to the photovoltaic effect, which is applied on patches 2 and 3 through the conducting material. As discussed previously, the actuator patches 1 and 2 on the bottom surface deform in extension, while the actuator patch 3 on the top surface deforms in contraction. In summary, positive membrane and bending control force can be induced. In view of $h_{a} \ll h \ll R$ and the above analysis, it is seen that the membrane forces induced 


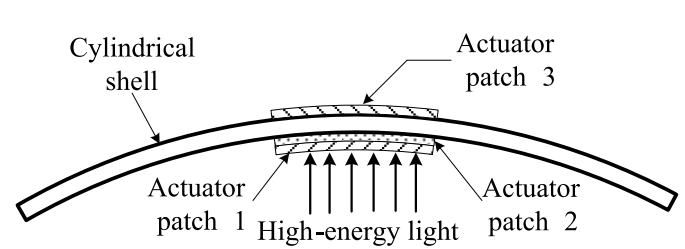

(a)

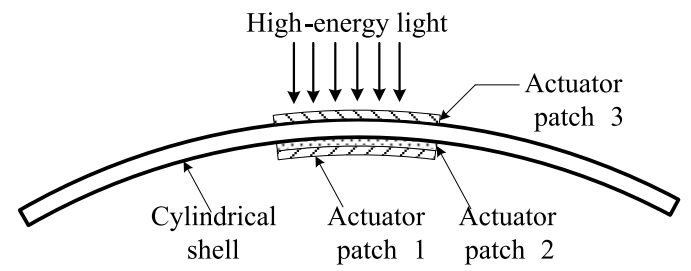

(b)

Fig. 5. Illumination way: (a) the bottom illumination; (b) the upper illumination.

by the actuator patches 1 and 2 are approximately equal to the membrane forces induced by the actuator patch 3 but their signs are opposite, so the resultant circumferential mode control $F_{m n}^{c}$ could be rewritten as

$$
F_{m n}^{c} \approx-\left(\tilde{M}_{\psi m n}+\tilde{N}_{\psi m n}+\tilde{M}_{\psi m n}+\tilde{N}_{\psi m n}+\tilde{M}_{\psi m n}-\tilde{N}_{\psi m n}\right) \bar{S}=-\left(3 \tilde{M}_{\psi m n}+\tilde{N}_{\psi m n}\right) \bar{S}
$$

When the cylindrical shell oscillates upward, light should be applied to the top actuator, and this top actuator deforms in extension, while the bottom actuators deform in contraction. In sum, negative membrane and bending control force can be induced. The resultant circumferential-mode control $F_{m n}^{c}$ could be rewritten as

$$
F_{m n}^{c} \approx-\left(-M_{\psi m n}-\tilde{N}_{\psi m n}-\tilde{M}_{\psi m n}-\tilde{N}_{\psi m n}-\tilde{M}_{\psi m n}+\tilde{N}_{\psi m n}\right) \bar{S}=\left(3 \tilde{M}_{\psi m n}+\tilde{N}_{\psi m n}\right) \bar{S}
$$

Compared with the existing photostrictive actuator configuration [17-20], the proposed actuator configuration can not only produce a positive membrane control force but also a negative membrane control force. It is thus clear that, based on the proposed actuator configuration, the positive and negative modal control force can be efficiently generated to suppress vibration by changing the illumination direction. In summary, it is a prominent advantage for the multi-layer actuator configuration to produce the positive and negative membrane control force.

\section{Independent modal variable structure fuzzy active control algorithm}

\subsection{Sliding surface design}

As mentioned previously, the paired photostrictive actuators are respectively placed on the top and bottom surfaces of the cylindrical shell. By activating the light sources alternatively, both positive and negative control actions can be generated. It is apparent that the alternate mode of light illumination is important. In this paper, light switching function is taken as the following linear function [27,32]

$$
s=\lambda \eta_{m n}+\dot{\eta}_{m n}
$$

where $\lambda$ is a positive constant, which is simply the slope of the switching surface. When the light illumination is alternatively varied with the sign of the switching function, the modal control force $F_{m n}^{c}$ is rewritten as

$$
F_{m n}^{c}=-\operatorname{sign}(s) \cdot\left(3 \tilde{M}_{\psi m n}+\tilde{N}_{\psi m n}\right) \cdot \bar{S}(t)
$$

where $\operatorname{sign}(\cdot)$ is a signum function. In this paper, it is assumed that the remnant strain and electric field of actuators can disappear immediately when light direction is changed [9,20]. With reference to Eq. (12), it can be seen that the switching function is determined by parameter $\lambda$ only. Therefore, how to determine the value of $\lambda$ is the next issue to be considered.

According to the variable structure control theory, $\lambda$ must be chosen in such a way that the existence and the reaching of the sliding mode are guaranteed. The mathematical form of this condition can be stated as

$$
s \dot{s}<0 \text { or } \begin{cases}\dot{s}<0 & \text { if } s>0 \\ \dot{s}>0 & \text { if } s<0\end{cases}
$$




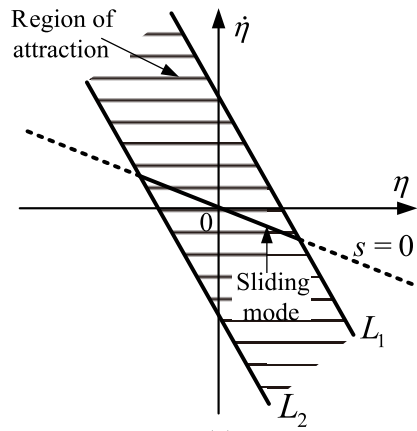

(a)

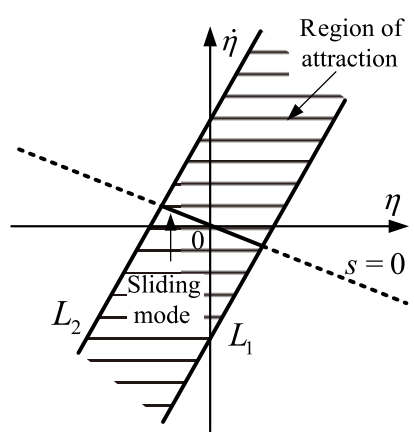

(b)

Fig. 6. Sliding mode (a) $2 \zeta_{m n} \omega_{m n}-\lambda>0$, (b) $2 \zeta_{m n} \omega_{m n}-\lambda<0$.

Take the derivative of sliding surface with respect to time, thus

$$
\dot{s}=\lambda \dot{\eta}_{m n}+\ddot{\eta}_{m n}
$$

Substituting Eqs (3) and (13) into Eq. (15), then

$$
\left\{\begin{array}{lll}
\dot{s}=\lambda \dot{\eta}_{m n}+\ddot{\eta}_{m n}=-\left(2 \zeta_{m n} \omega_{m n}-\lambda\right) \dot{\eta}_{m n}-\omega_{m n}^{2} \eta_{m n}+\left|F_{m n}^{c}\right|>0 & \text { when } s<0 \\
\dot{s}=\lambda \dot{\eta}_{m n}+\ddot{\eta}_{m n}=-\left(2 \zeta_{m n} \omega_{m n}-\lambda\right) \dot{\eta}_{m n}-\omega_{m n}^{2} \eta_{m n}-\left|F_{m n}^{c}\right|<0 & \text { when } s>0
\end{array}\right.
$$

Equation (16) shows that only if the system state trajectory in phase plane moves into the region between the two lines $L_{1}$ and $L_{2}$ (Fig. 6), the reaching conditions could be satisfied. This part of the area is known as the region of attraction. The lines $L_{1}$ and $L_{2}$ are formulated as

$$
\begin{aligned}
& L_{1}: \omega_{m n}^{2} \eta_{m n}+\left(2 \zeta_{m n} \omega_{m n}-\lambda\right) \dot{\eta}_{m n}=\left|F_{m n}^{c}\right| \\
& L_{2}: \omega_{m n}^{2} \eta_{m n}+\left(2 \zeta_{m n} \omega_{m n}-\lambda\right) \dot{\eta}_{m n}=-\left|F_{m n}^{c}\right|
\end{aligned}
$$

As shown in Fig. 6, the switching line $s=0$ located in the region of attraction is known as the sliding mode. With ideally switching control force $F_{m n}^{c}$, the system trajectory could reach the sliding area in finite time. Once the system trajectory slides toward the origin along the switching line, the vibration will be suppressed [33]. In the sliding mode area, system states satisfy $s=\lambda \eta_{m n}+\dot{\eta}_{m n}=0$, that is, $\dot{\eta}_{m n}=-\lambda \eta_{m n}$. It is thus clear that $\lambda>0$ guarantees the stability of sliding mode motion. At the same time, the parameter $\lambda$ directly determines the sliding speed and influences the range of the region of attraction. Thereby the length of the sliding mode area $l$ is written as

$$
l=\frac{2\left|F_{m n}^{c}\right| \sqrt{1+\lambda^{2}}}{\left|\omega_{m n}^{2}-2 \zeta_{m n} \omega_{m n} c+\lambda^{2}\right|}
$$

From Eq. (18), it can be seen that the length of the sliding mode area is proportional to the modal control force $\left|F_{m n}^{c}\right|$, thus increasing the intensity of the modal control force is beneficial. For the flexible structure, $0 \leqslant \zeta_{m n}<1$, the length of the sliding mode is limited. When $\lambda$ equal to the product of $\zeta_{m n}$ and $\omega_{m n}$, the maximum value of the length of the sliding mode, which is most favorable to the vibration suppression, is obtained. Thus the expression of $s$ can be rewritten as

$$
s=\zeta_{m n} \omega_{m n} \eta_{m n}+\dot{\eta}_{m n}
$$

\subsection{Light intensity self-tuning fuzzy control design}

Fuzzy control (FC) has supplanted conventional technologies in many applications. One major feature of fuzzy control is its ability to express the amount of ambiguity in human thinking. Thus, when the mathematical model of the process does not exist, or exists but with uncertainties, FC is an alternative way to deal with the unknown process. The designed light intensity self-tuning fuzzy active controller is shown in Fig. 7. 


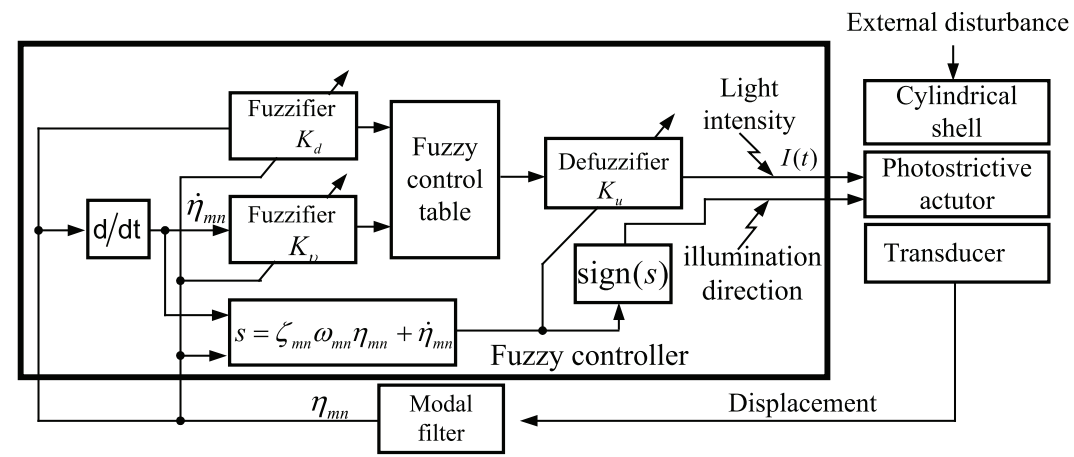

Fig. 7. Structure diagram of fuzzy controller.

The center of the shell ( $x=L / 2$ and $\left.\Psi=\Psi^{*} / 2\right)$ is used as the reference point to evaluate the control effectiveness. A laser Doppler vibrometer remotely measures the displacement of this reference point, which is used as the feedback reference in a closed-loop control system. A modal filter is used to obtain the designated modal displacement sensed by the laser vibrometer. The designated modal displacement and its derivative are chosen as the inputs to fuzzy controller and are used to construct the sliding mode function. Here, the sign of the sliding mode function is taken as an index to decide which actuator should be irradiated. The designated modal displacement and velocity are multiplied by the scale factors $K_{d}$ and $K_{v}$, respectively. The fuzzy output is determined from a look-up table. This fuzzy output is then assigned a numerical value which is multiplied by $K_{u}$ to obtain the desired light intensity $I(t)$.

The system response only changes near the fuzzy linguistic value "ZO" when the displacement $\eta_{m n}$ becomes very small in vibration. Meanwhile, the other fuzzy linguistic values clearly do not work in description and resolution for the displacement at the moment. Ideally, when the displacement decreases, fuzzy partition should also be able to fully describe and analyze small displacement change, so as to improve the control system resolution capacity in the small range, it is required to real-time tuning for scaling factors. In the meantime, for the sake of most possibly increasing the modal control force, tuning law for fuzzifier factors, $K_{d}$ and $K_{v}$, are selected as

$$
K_{d}=\frac{3}{\eta_{m n}}, K_{v}=\frac{3}{\eta_{m n} \omega_{m n}} \text { when } s=\zeta_{m n} \omega_{m n} \eta_{m n}+\dot{\eta}_{m n}=0
$$

Fuzzy scaling factors, $K_{d}$ and $K_{v}$, are responsible for mapping the inputs of displacement $\eta_{m n}$ and velocity $\dot{\eta}_{m n}$, to the universes of discourse $[-3,3]$.

Since vibration has been basically suppressed in the later stage of the vibration control, there is no need to impose control force, and then the light intensity should be reduced to zero. Therefore, tuning law for defuzzifier factors $K_{u}$ is selected as

$$
K_{u}=\frac{|s|}{|s|+\delta} \cdot \frac{I_{\max }}{3}
$$

where $|s|$ is the absolute value of switching function $s=\zeta_{m n} \omega_{m n} \eta_{m n}+\dot{\eta}_{m n}, I_{\max }$ is the provided maximum of the light intensity, $\delta$ is small positive constant.

Fuzzy controller is designed as follows:

\section{(1) Selecting linguistic variables and membership functions}

2-D fuzzy controller is adopted, namely double input and single output fuzzy controller. The chosen inputs of the fuzzy controller are the modal displacement $\eta_{m n}$ and velocity $\dot{\eta}_{m n}$. The chosen output is the light intensity $I(t)$ irradiated on the photostrictive actuators. Considering that light intensity $I(t)$ is always positive, the output is defined on the universe of discourse [0,3]. Accordingly, the set of linguistic values for the inputs are $\{\mathrm{NL}, \mathrm{NM}, \mathrm{NS}, \mathrm{ZO}$, PS, PM, PL $\}$, and one of the output is $\{$ ZO, PS, PM, PL $\}$. Here, NL, NM, NS, ZO, PS, PM, and PL refer to negative large, negative medium, negative small, zero, positive small, positive medium, and positive large, respectively.

The membership function is used to describe the fuzziness of the fuzzy set elements. Its choice directly affects the accuracy of the fuzzy problem solving. In this paper, the triangular membership functions shown in Figs 8(a) and (b) are used, which fuzzify the set elements on the universe of discourse. 
Table 1

Fuzzy control rules

\begin{tabular}{lccccccc}
\hline$\eta$ & $\mathrm{NL}$ & $\mathrm{NM}$ & $\mathrm{NS}$ & $\mathrm{ZO}$ & $\mathrm{PS}$ & $\mathrm{PM}$ & $\mathrm{PL}$ \\
\hline $\mathrm{NL}$ & $P L$ & $P L$ & $P L$ & $Z O$ & $P S$ & $P S$ & $\mathrm{PS}$ \\
$\mathrm{NM}$ & $P L$ & $P L$ & $P L$ & $Z O$ & $P S$ & $P S$ & $\mathrm{PM}$ \\
$\mathrm{NS}$ & $P L$ & $P L$ & $P L$ & $Z O$ & $P S$ & $P M$ & $\mathrm{PL}$ \\
$\mathrm{ZO}$ & $P L$ & $P L$ & $P L$ & $Z O$ & $P L$ & $P L$ & PL \\
$\mathrm{PS}$ & $P L$ & $P M$ & $P S$ & $Z O$ & $P L$ & $P L$ & $\mathrm{PL}$ \\
$\mathrm{PM}$ & $P M$ & $P S$ & $P S$ & $Z O$ & $P L$ & $P L$ & PL \\
$\mathrm{PL}$ & $\mathrm{PS}$ & $\mathrm{PS}$ & $\mathrm{PS}$ & $\mathrm{ZO}$ & $\mathrm{PL}$ & $\mathrm{PL}$ & $\mathrm{PL}$ \\
\hline
\end{tabular}

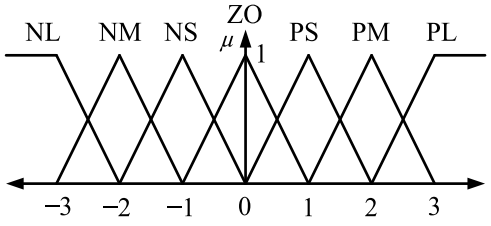

(a)

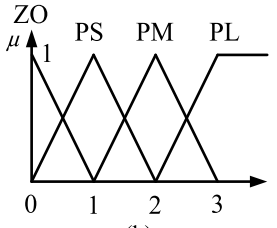

(b)

Fig. 8. Membership functions of the inputs and output: (a) the inputs, (b) the output.

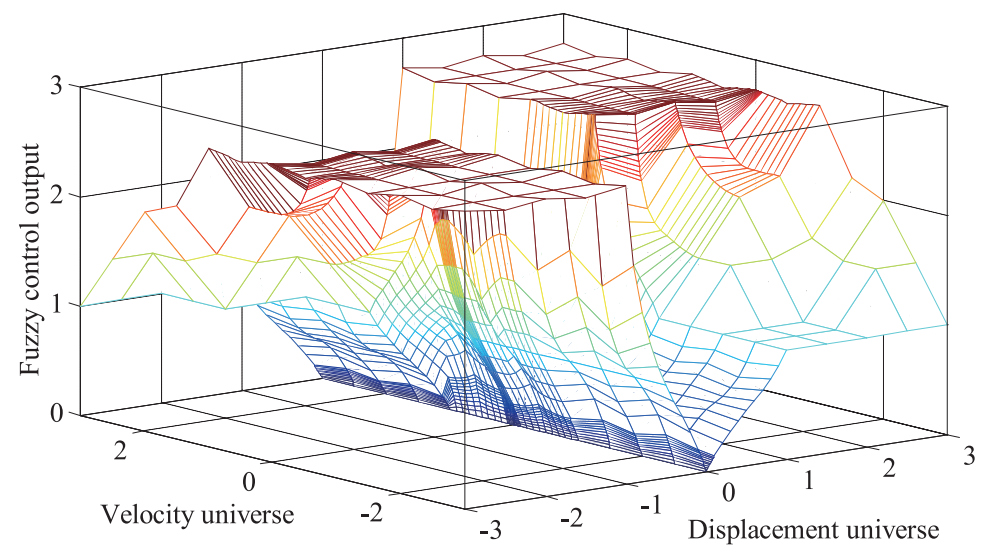

Fig. 9. The control surface.

\section{(2) The establishment of the fuzzy control rules}

According to the fuzzy control rules, fuzzy output can be inferred from the input of the fuzzy. Usually, fuzzy control rules are based on the actual control experience. With reference to the history of vibration response, it is observed that, when $\eta_{m n} \leqslant 0$ and $\dot{\eta}_{m n}<0$, control force $F_{m n}^{c}$ imposed on the cylindrical shell should be large. For the photostrictive actuator, in order to produce a large control force $F_{m n}^{c}$, control light intensity have to be large, corresponding to the fuzzy linguistic value PL. Similarly, when $\eta_{m n} \geqslant 0$ and $\dot{\eta}_{m n}>0$, one of control light intensity should also be PL. However, when $\eta_{m n}<0$ and $\dot{\eta}_{m n}>0$ or $\eta_{m n}>0$ and $\dot{\eta}_{m n}<0$, there is no need to impose large control force on the cylindrical shell, so fuzzy linguistic value of control light intensity should be $\mathrm{ZO}$, PS or PM. Through summing up the above-mentioned experience, the fuzzy control rules are shown in Table 1.

\section{(3) Defuzzification}

The resulting fuzzy set must be converted to a single crisp value in order to form a control signal to the plant. There are three common methods: COG (centre of gravity), BOA (bisector of area), and MOM (mean of maxima). In this paper, COG method is adopted. The resulting defuzzified value is

$$
u_{C O G}=\frac{\int_{X} \mu_{x}(x) x \mathrm{~d} x}{\int_{X} \mu_{x}(x) \mathrm{d} x}
$$

where $x$ is a point in the universe $X$ of the resulting fuzzy set, and $\mu_{x}(x)$ is the membership of the resulting conclusion set.

\section{(4) Building fuzzy control table}

The basic idea of fuzzy control table is shown as follows. Firstly, fuzzy control table is built by means of inferring all possible control actions offline with discrete premise universes before putting the controller into operation. With two controller inputs and one output, the table is a 2-D lookup table. Secondly, fuzzy control table is stored 


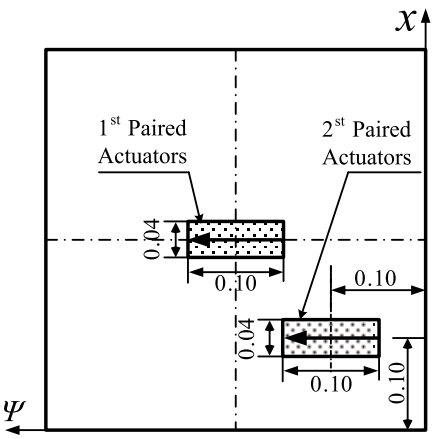

(a)

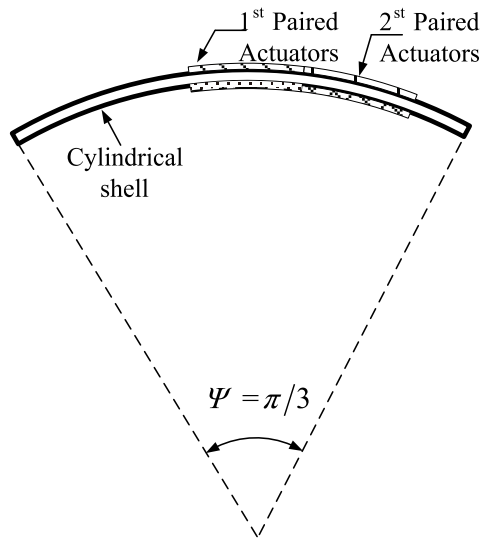

(b)

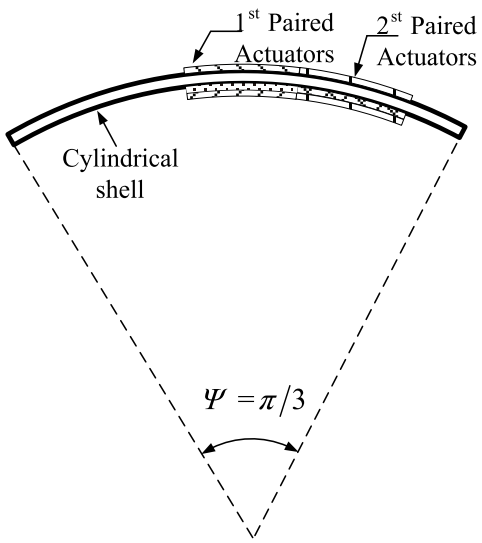

(c)

Fig. 10. Configuration of photostrictive actuators laminated on the cylindrical shell: (a) top view; (b) side view for the existing actuator configuration; (c) side view for the proposed actuator configuration.

in computer memory. Finally, according to the scaling values of the sampling modal displacement $\eta_{m n}$ and velocity $\dot{\eta}_{m n}$, the corresponding fuzzy output is derived by looking up the control table. The desired light intensity $I(t)$ is obtained via multipling the fuzzy output value by $K_{u}$. The pre-calculation improves execution speed, as the runtime inference is reduced to a table lookup.

In this paper, it is assumed that there is a resolution of 47 points in each universe, so the table holds $47^{2}$ elements. In order to improving the control effect, the choice of 10 points is from the universe $[-3,-1]$ and $[1,3]$ with step of 0.5 , the choice of 18 points is from the universe $[-0.9,-0.1]$ and $[0.1,0.9]$ with step of 0.1 , and the choice of 19 points is from the universe $[-0.09,0.09]$ with step of 0.01 . That is to say, the smaller the control input value, the higher the resolution. Since the fuzzy control table holds too many elements, it is not given in detail. Figure 9 illustrates a nonlinear control surface include $47^{2}$ points, of which every four point constitute a rectangular plane. In addition, Fig. 9 suggests that fuzzy control table is consistent with the fuzzy control rules.

\subsection{Chattering reduction}

The phenomenon of some oscillations in some vicinity of the switching surface is called chattering problem. Due to physical limitations in real-world systems, directly applying the control law showed in Eq. (13) usually leads to chattering problem. Chattering is undesirable, since it may excite high frequency modes neglected in the course of modeling, which degrades the performance of the system and may even lead to instability. Chattering must be reduced for the controller to perform properly. This can be achieved by smoothing out the control discontinuity in a thin boundary layer [26,27,32]. In this paper, the following continuous function $\Delta(s)$ is used to approximate the sign function $\operatorname{sign}(s)$ :

$$
\Delta(s)=\frac{s}{|s|+\delta} \quad(\delta>0)
$$

\section{Case studies}

On the basis of theoretical analysis presented in the preceding sections, there are two case studies carried out: 1) the actuation effectiveness analysis of the existing actuator configurations [17,20] and the proposed one; and 2) two variable light control algorithms, namely a) the proposed independent modal variable structure fuzzy active control and b) the proportional velocity feedback control used in the literatures [9,20], are implemented and their control effectiveness are compared. With reference to the literature [20], the cylindrical shell studied here is made of Plexiglas $\left(Y=3.1 \times 10^{9} \mathrm{~N} / \mathrm{m}^{2}, \mu=0.35\right.$, and $\left.\rho=1190 \mathrm{~kg} / \mathrm{m}^{3}\right)$. Dimensions of the cylindrical shell are: 


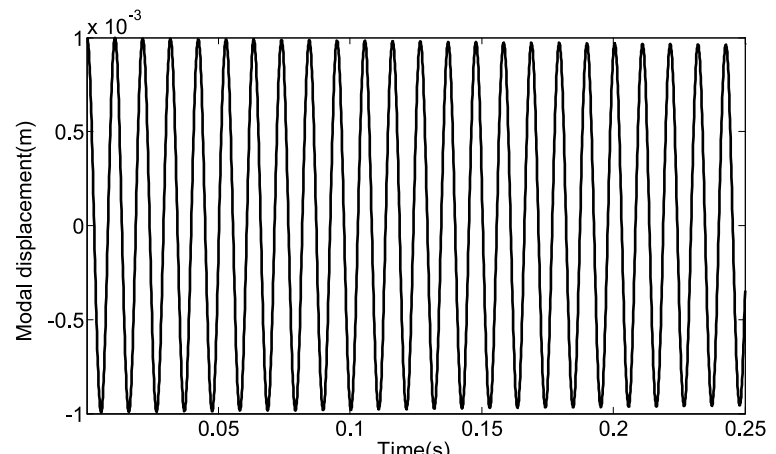

(a)

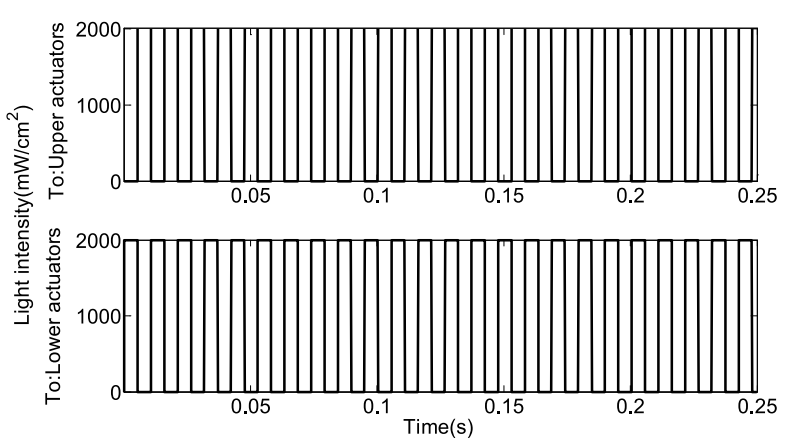

(c)

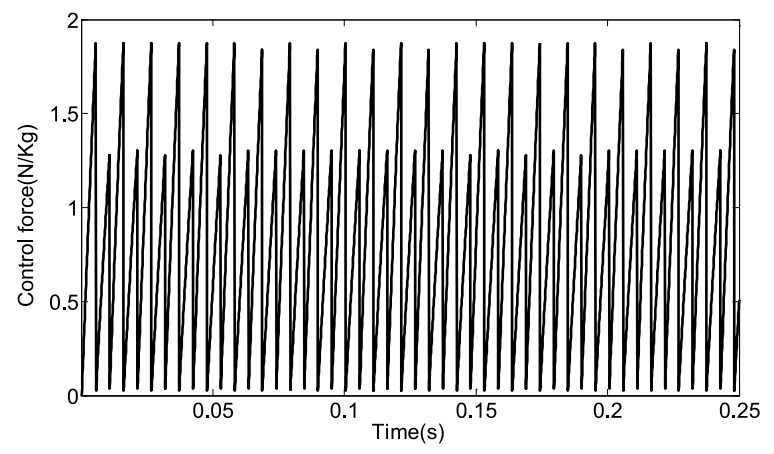

(b)

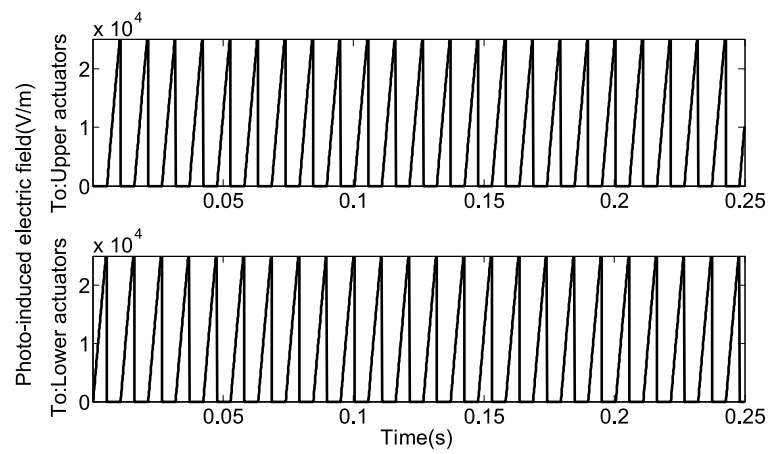

(d)

Fig. 11. Time histories for the existing actuator configuration [20].

$L=0.4 \mathrm{~m}, L_{\Psi}=0.4 \mathrm{~m}, \Psi=\pi / 3$, and $h=1.6 \times 10^{-3} \mathrm{~m}$. All PLZT actuators, with the same dimension: $0.1 \times 0.04 \times 0.0001 \mathrm{~m}^{3}$, are perfectly bonded to the shell, and the PLZT material properties are listed in Table A1. And the corner coordinates or locations of the two paired actuators can be defined with the dimensions labeled in Fig. 10(a). The $(1,3)$ mode $(94.83 \mathrm{~Hz})$ was taken as the sample mode evaluated in closed-loop controls. In order to observe the time histories of displacement responses, an initial modal displacement of $0.001 \mathrm{~m}$ is imposed to (1, 3 ) mode. However, since the modal control force varies with time, it is difficult to obtain an analytical solution of the modal equation. Thus, a classical numerical method, Newmark- $\beta$ method was adopted to estimate discrete time responses.

\subsection{Actuation effectiveness analysis of the existing and the proposed actuator configurations}

In this section, the actuation effectiveness of the existing actuator configuration $[17,20]$ and the proposed actuator configurations is studied and compared. As the location and size of the actuators strongly influence the controllability properties of the actuator configuration [17,20], it is assumed that the actuators' location and size of the existing actuator configuration is exactly the same as that of the proposed actuator configuration, as showed in Fig. 10(a). The side views of two configurations are showed in Figs 10(b) and (c), respectively. In order to show clearly the actuation ability of the actuator configuration, the structural damping is not taken into account, and the photodeformationinduced active damping exclusively contributes to the vibration control. In addition, to ensure that the exciting signal applied to two actuator configuration is identical, the constant light intensity control is used. In the constant light intensity control, the amplitude of the light intensity is constant and the light intensity $I(t)$ is defined as follows [9, 17,20]:

$$
I(t)=G\left[\max \left|\dot{\eta}_{m n}(t)\right|\right]
$$

where $G$ is the feedback gain. The direction of the light is alternatively changed according to the velocity direction. The time histories for the existing actuator configuration are presented in Figs 11(a)-(d), respectively. As seen in 


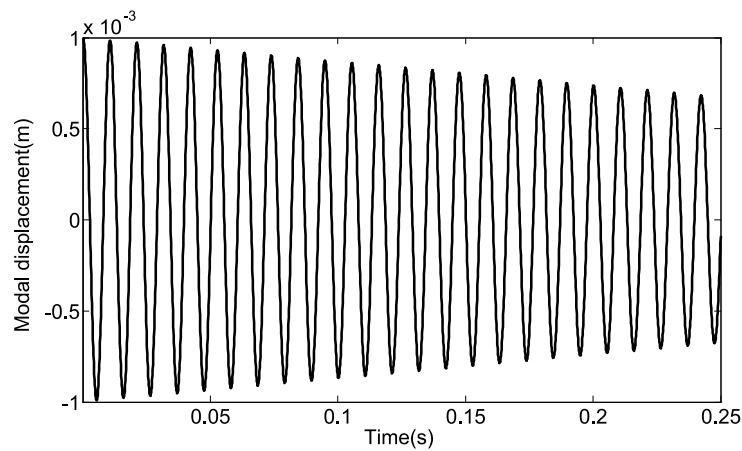

(a)

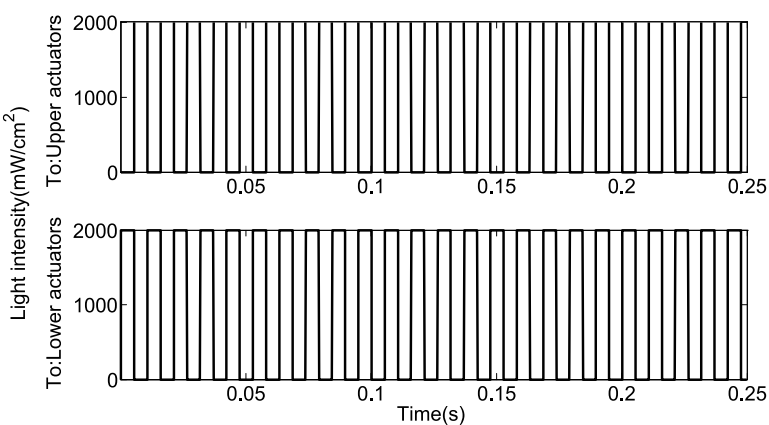

(c)

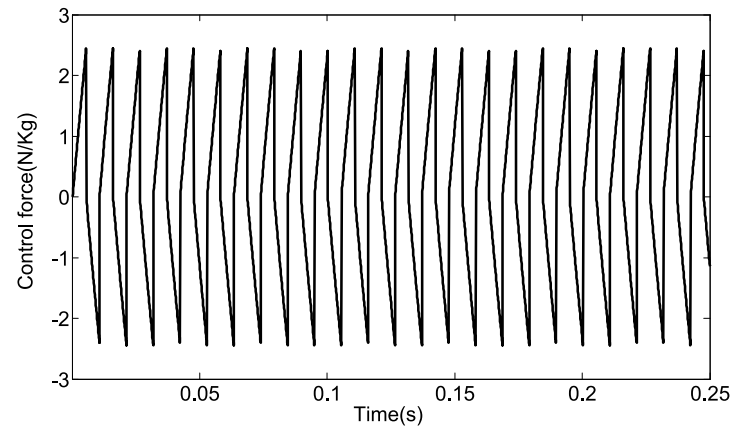

(b)

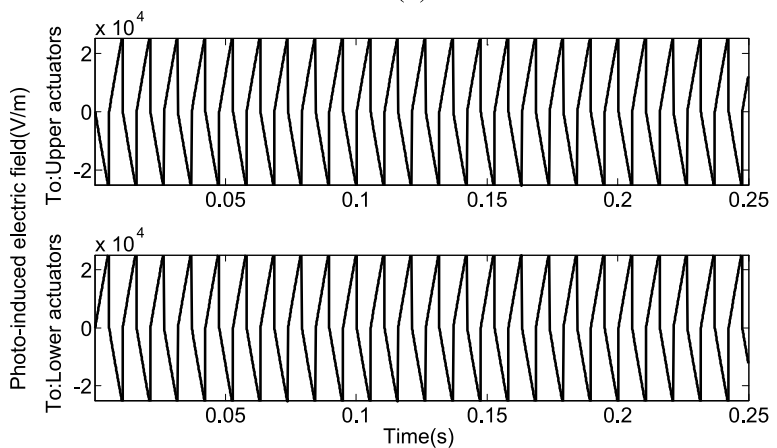

(d)

Fig. 12. Time histories for the proposed actuator configuration.

Fig. 11(a), the amplitude of the shell does not obviously decay, so it is impracticable to suppress vibration with the existing actuator configuration. The reason is that only the positive control force is induced, as showed in Fig. 11(b). A simple solution to this problem is half-cycle control, that is, the light can be applied on the actuators only when the positive control force is needed in vibration control, but this method undoubtedly reduces the efficiency of vibration suppression comparing with the full cycle control.

A novel multi-layer actuator configuration is proposed to remedy the deficiency for the existing actuator configuration. The time histories with the proposed actuator configuration are presented in Figs 12(a)-(d), respectively. As seen in Fig. 12(a), the amplitude of the shell obviously decay and the proposed actuator configuration could suppress the vibration effectively. Figure 12(b) shows that the proposed actuator configurationinduces both positive and negative control force. Figure 12(c) illustrates time histories of control light intensity applied on upper and lower actuators, which are the same as Fig. 11(c). Figure 12(d) illustrates photo-induced electric fields for upper and lower actuators with the proposed actuator configuration are positive and negative alternating changing, which is different obviously from that of Fig. 11(d).

\subsection{Control algorithms}

To give the further comparison, the variable structure fuzzy active control and the proportional velocity feedback control will be used to suppress the simply supported cylindrical shell vibration laminated with photostrictive actuators, respectively. As a variable light control scheme, the proportional velocity feedback control has been studied in the literatures $[9,20]$. In the proportional velocity feedback control, the light intensity is proportional to the vibration velocity, and the amplitude of light intensity fluctuates with the velocity. Note that the light intensity is always positive. Thus, the control light intensity applied to the actuator system can be written as [9,20]:

$$
I\left(t_{j}\right)=G\left|\dot{\eta}_{m n}\left(t_{j-1}\right)\right|
$$




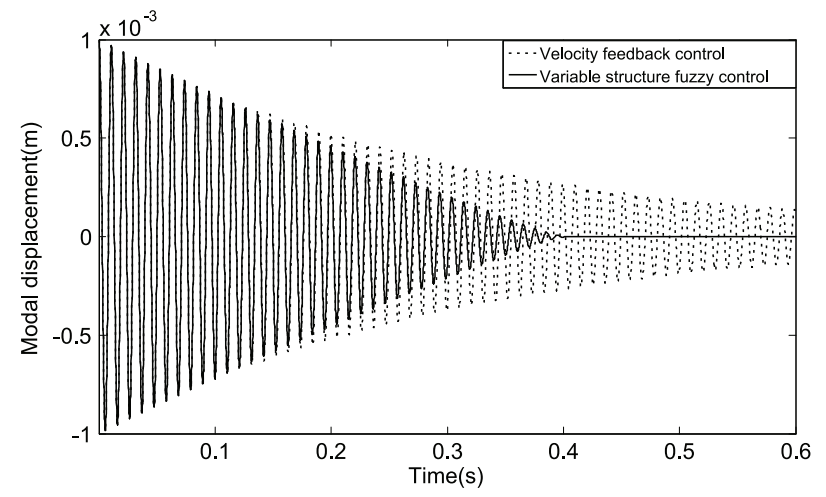

Fig. 13. Time histories of displacement response with the velocity feedback control and variable structure fuzzy control.

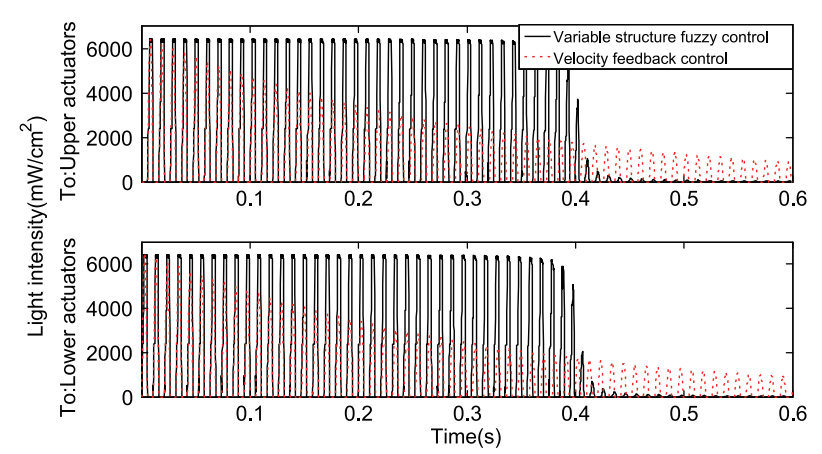

Fig. 15. Time histories of light intensity.

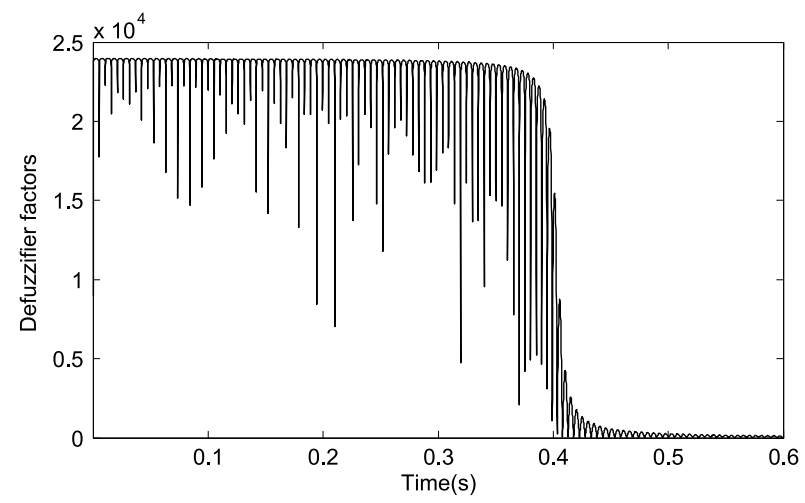

Fig. 17. Time history of self-tuning defuzzifier factors $K_{u}$.

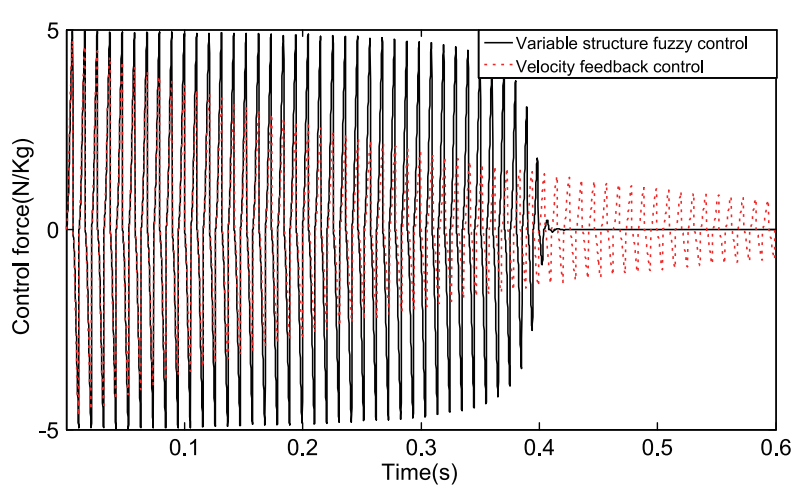

Fig. 14. Time histories of control force.

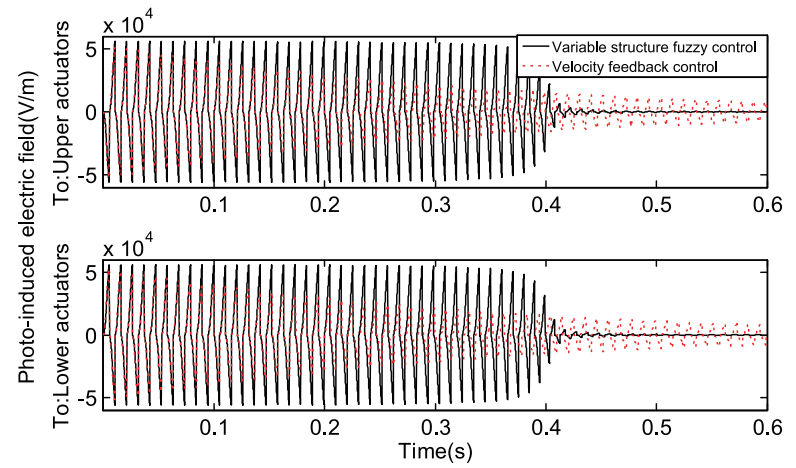

Fig. 16. Time histories of photo-induced electric field.

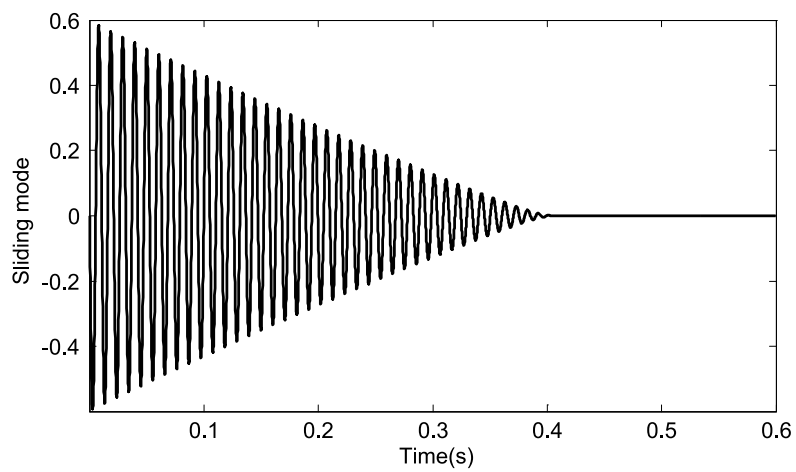

Fig. 18. Time history of sliding mode.

where $G$ is the light intensity gain. However, as a result of the complex nonlinear and time-variant behaviors being exist in photostrictive actuator, the control effect of proportional velocity feedback is not very ideal.

Noting that the designed optical switching surface is identical with the switching surface of the velocity, the damping ratio $\xi_{13}$ is set as 0.001 in this study. With reference to Eq. (19), the light switching function is $s=0.59583 \eta_{13}+\dot{\eta}_{13}$. Applying the proposed chattering reduction scheme, the parameter $\delta$ takes a small positive constant 0.001 .

Based on the proportional velocity feedback control and the proposed control algorithms, time histories of modal displacement, control force, control light intensity, and photo-induced electric field are presented in Figs 13-16, 


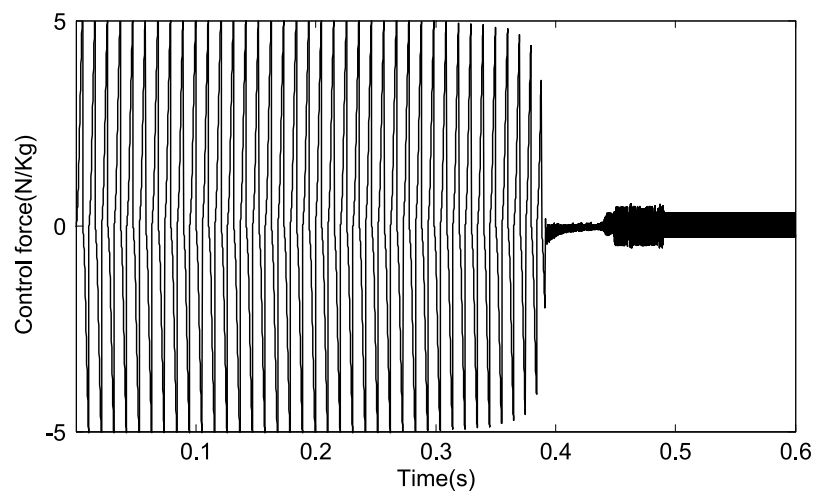

Fig. 19. Time history of control force without chattering reduction.

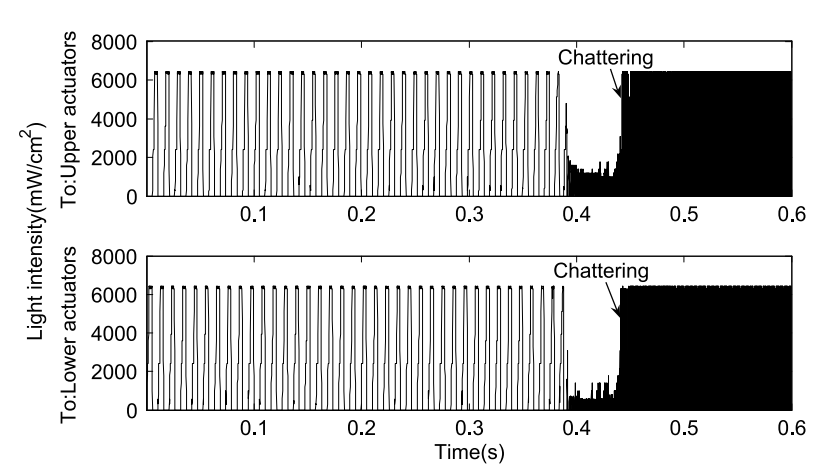

Fig. 20. Time histories of light intensity without chattering reduction.

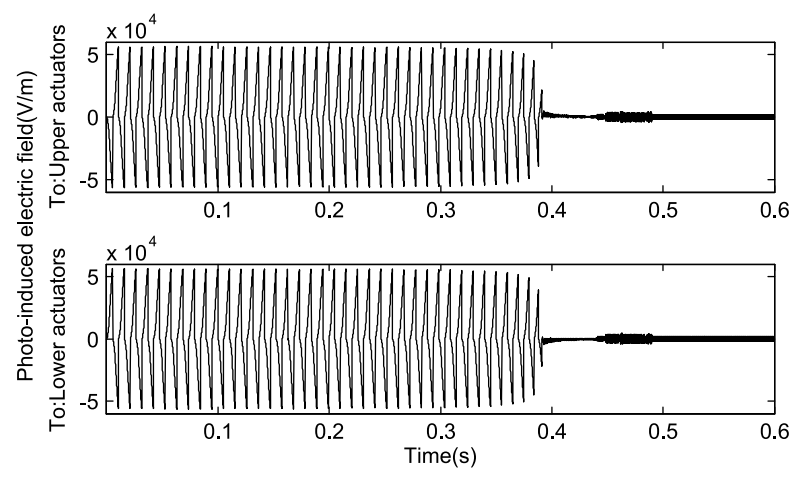

Fig. 21. Time histories of photo-induced electric field without chattering reduction.

respectively. From Fig. 13, it is obviously seen that the proposed control algorithm provides better control responses than the proportional velocity feedback control. As seen in Fig. 15, due to the use of the proposed self-tuning law for fuzzifier factors $K_{d}$ and $K_{v}$, the larger light intensity is applied to the top and bottom actuators in the earlier stages of the vibration control $(0 \sim 0.4 \mathrm{~s})$. At the same time, it is also shown that, when the vibration is gradually suppressed, and system state approaches on the sliding surface, at this time $s \approx 0$, the light intensity is reduced to zero according to the tuning law for defuzzifier factors $K_{u}$. Figure 14 shows that the modal control force with the proposed control algorithm is larger than that with the proportional velocity feedback in the earlier stage of the vibration control $(0 \sim 0.4 \mathrm{~s})$, and it is gradually reduced to zero with the light intensity being reduced to zero in the later stage. Figure 16 shows that the amplitude of photo-induced electric field with the proposed control algorithm is much larger than that of the proportional velocity feedback control. Figures 17 and 18 illustrate the varying curves of the defuzzifier factors $K_{u}$ and sliding mode over time with the proposed control algorithm, respectively. Figure 18 suggests that the sliding mode is asymptotic stability and finally approaches to zero.

The time history of control light intensity without chattering reduction is presented in Fig. 20. Comparing Fig. 20 with Fig. 15, it is suggested that chattering for variable structure control is reduced by the introduction of the proposed chattering reduction method. As shown in Fig. 21, since there is the chattering, the switching of light direction is very fast; accordingly, the photo-induced electric field is very small. In the meantime, the control force is very small, which is presented in Fig. 19.

\section{Conclusions}

Non-contact control and precision actuation using photostrictive actuators offer many advantages over the conventional hard-wired actuator systems. Such optically triggered, non-contact actuator provides a type of novel alternative 
actuation for active vibration control of aerospace vehicle. In this paper, the photostrictive actuators are applied to vibration control of thin cylindrical shell. To cope with the problem that the existing actuator configuration can not generate positive and negative membrane force, a novel multi-layer actuator configuration is explored. Then, the independent modal vibration control equations of cylindrical thin shells laminated with the proposed actuator configuration are established. The variable structure self-adjusting parameters fuzzy control algorithm of light intensity is investigated, in which off-line fuzzy control table is adopted. The optimal switching surface is derived to increase the range of sliding mode to facilitate the vibration suppression. To reduce the variable structure control chattering, the continuous function $\Delta(s)$ is used to replace sign function $\operatorname{sign}(s)$ for smoothing out the control discontinuity in a thin boundary layer.

In case studies, the actuation effectiveness of the existing and proposed actuator configurations is analyzed and compared. Two control algorithms are implemented and compared. Analytical and simulation results suggest the following:

1) The proposed multi-layer actuator configuration could generate positive and negative membrane force. Compared with the existing actuator configuration, it has an obvious advantage in achieving non-contact active vibration control of shell structures. It provides a new actuator configuration for the active vibration control of this kind of flexible structure.

2) Because of the complex nonlinear and time-variant behaviors of photostrictive actuators, the proportional velocity feedback control can not do as well as expected. Comparatively speaking, variable structure fuzzy control algorithm could give the better control effect. Due to the self-adjusting parameters scheme applied in the proposed control algorithm, the larger modal control force is produced, and thus the vibration is suppressed rapidly. Once system trajectory approaches onto the sliding surface, the modal control force applied to the shell system could approach near zero. Due to the introduction of the proposed chattering reduction scheme, chattering for variable structure control is significantly reduced, and the control quality of system is improved.

3) As a variable light control scheme, the proposed control algorithm is also suitable for active vibration control of other flexible structure laminated with photostrictive actuators.

Finally, note that this study is carried out based on analytical solutions, which would need to be further verified by laboratory experiments.

\section{Acknowledgments}

This research was supported by a grant from the Chinese Natural Science Foundation (No. 10872090, 50830201), Nanjing University of Aeronautics and Astronautics Special Research Projects (No. NJ2010011) and the Project Funded by the Priority Academic Program Development of Jiangsu Higher Education Institutions.

\section{Appendix}

Table A1

Material properties of photostrictive actuators

\begin{tabular}{ccl}
\hline Parameter & Value & \multicolumn{1}{c}{ Property } \\
\hline$E_{s}$ & $2.43 \times 10^{5} \mathrm{~V} / \mathrm{m}$ & Saturated electric field \\
$Y_{a}$ & $6.3 \times 10^{10} \mathrm{~N} / \mathrm{m}^{2}$ & Young's modulus \\
$\alpha$ & $2.772 \times 10^{-3} \mathrm{~m}^{2} /(\mathrm{ws})$ & Optical actuator constant \\
$\beta$ & $0.01 \mathrm{~V} / \mathrm{s}$ & Voltage leakage constant \\
$P$ & $0.23 \times 10^{3} \mathrm{~cm}^{2} / \mathrm{s}$ & Power of absorbed heat \\
$d_{33}$ & $1.79 \times 10^{-10} \mathrm{~m} / \mathrm{V}$ & Piezoelectric strain constant \\
$H$ & $16 \mathrm{~W} /{ }^{\circ} \mathrm{C}$ & Heat capacity \\
$\gamma$ & $0.915 \mathrm{w} /{ }^{\circ} \mathrm{C} \mathrm{s}$ & Heat-transfer rate \\
$\lambda$ & $6.8086 \times 10^{4} \mathrm{~N} / \mathrm{m}^{2 \circ} \mathrm{C}$ & Stress-temperature constant \\
$P_{n}$ & $0.25 \times 10^{-4} \mathrm{C} / \mathrm{m}^{2 \circ} \mathrm{C}$ & Pyroelectric constant \\
$\varepsilon$ & $1.65 \times 10^{-8} \mathrm{~F} / \mathrm{m}$ & Electric permittivity \\
\hline
\end{tabular}




\section{References}

[1] E.F. Crawley, Intelligent structures for aerospace: A technology overview and assessment, AIAA Journal 32 (1994), $1689-1699$.

[2] C. Chee, L. Tong and G.P. Steven, A review on the modeling of piezoelectric sensors and actuators incorporated in intelligent structures, Journal of Intelligent Material Systems and Structures 9 (1998), 13-19.

[3] H.S. Tzou, H.-J. Lee and S.M. Arnold, Smart materials, precision sensors/actuators, smart structures and structronic systems, Mechanics of Advanced Materials and Structures 11 (2004), 367-393.

[4] T. Fukuda, S. Hattori, F. Arai, H. Matsuura, T. Hiramatsu, Y. Ikeda and A. Maekawa, Characteristics of optical actuator-servomechanisms using bimorph optical piezoelectric actuator, IEEE Robotics and Automation Conference 2 (1993), 618-623.

[5] T. Fukuda, S. Hattori, F. Arai and H. Nakamura, Performance improvement of optical actuator by double side irradiation, IEEE Transactions on Industrial Electronics 42 (1995), 455-461.

[6] K. Uchino, Photostrictive actuator, in: IEEE Ultrasonics Symposium, Honolulu, 1990, pp. 721-723.

[7] K. Uchino, New applications of photostrictive ferroics, Materials Research Innovations 1 (1997), $163-168$.

[8] P. Poosanaas, K. Tonooka and K. Uchino, Photostrictive actuators, Mechatronic 10 (2000), 467-487.

[9] B. Liu and H.S. Tzou, Distributed photostrictive actuation and opto-piezothermoelasticity applied to vibration control of plates, ASME Journal of Vibration and Acoust 120 (1998), 937-943.

[10] H.R. Shih and H.S. Tzou, Opto-piezothermoelastic constitutive modeling of a new 2-d photostrictive composite plate actuator, in: Proceedings of 2000 ASME International Mechanical Engineering Congress and Exposition, New York, USA 61 (2000), 1-8.

[11] H.S. Tzou and C.S. Chou, Nonlinear opto-electromechanics and photodeformation of optical actuators, Smart Materials and Structures 5 (1996), 230-235.

[12] Y. Morikawa and T. Nakada, Position control of PLZT bimorph-type optical actuator by on-off control, Proc of the 23rd Int Conf on Industrial Electronics, Control and Instrumentation (1997), 1403-1408.

[13] H.R. Shih and H.S. Tzou, Opto-electromechanical actuator patches for active vibration control of thin plates, 42th AIAA/ASME/ASCE/AHS Structures, Structural Dynamics and Materials Conference, Seattle, WA, Paper No. AIAA-2001-1556, 2001.

[14] H.R. Shih, H.S. Tzou and K. McLin, Optimal control of beam dynamics with segmented photostrictive actuators, in: 44th AIAA/ ASME/ASCE/AHS Structures, Structural Dynamics and Materials Conference, Norfolk, VA, Paper No. AIAA-2003-1648, 2003.

[15] H.R. Shih, H.S. Tzou and M. Saypuri, Structural vibration control using spatially configured opto-electromechanical actuator, Journal of Sound and Vibration 284 (2005), 361-78.

[16] L. Lei, S.P. Wang and F. Cao, Characteristics of photo-mechanical device using PLZT wafer, in: 2nd IEEE/ASME International Conference on Mechatronic and Embedded Systems and Applications (13-16 Aug 2006).

[17] H.R. Shih, R. Smith and H.S. Tzou, Photonic control of cylindrical shells with electro-optic photostrictive actuators, AIAA Journal 42 (2004), 341-347.

[18] H.R. Shih and H.S. Tzou, Photostrictive actuators for photonic control of shallow spherical shells, Journal of Smart Materials and Structures 16 (2007), 1712-1717.

[19] H.R. Shih, H.S. Tzou and W.L. Walters, Photonic control of flexible structures - application to a free-floating parabolic membrane shell, Journal of Smart Materials and Structures 18 (2009), 115019.

[20] X.J. Wang, H.H. Yue, J. Jiang, Z.Q. Deng and H.S. Tzou, Wireless active vibration control of thin cylindrical shells laminated with photostrictive actuators, Journal of Intelligent Material Systems and Structures 22 (2011), 337-357.

[21] H.H. Yue, G.L. Sun, Z.Q. Deng and H.S. Tzou, Distributed shell control with a new multi-DOF photostrictive actuator design, Journal of Sound and Vibration 329 (2010), 3647-3659.

[22] S.J. Zheng, Finite element simulation of wireless structural vibration control with photostrictive actuators, Science China Technological Sciences 55 (2012), 709-716.

[23] X.J. Wang, Research on photostrictive characteristics of PLZT and active control of photostrictive laminated thin shells of revolution, PhD Thesis, Harbin Institute of Technology, China, 2011.

[24] H.S. Tzou and J.P. Zhong, Spatially filtered vibration control of cylindrical shells, Shock and Vibration 3 (1996), $269-278$.

[25] V.I. Utkin, Variable structure system with sliding modes, IEEE Trans on Automatic Control AC-22 (1977), $212-222$.

[26] K.D. Young, V.I. Utkin and U. Ozguner, Control engineer's guide to sliding mode control, IEEE Trans On Control Systems Technology 7 (1999), 328-342

[27] M. Sharma and S.P. Singh, Fuzzy sliding mode control of plate vibrations, Shock and Vibration 17 (2010), 71-92.

[28] T. Takawa, T. Fukuda and K. Nakashima, Fuzzy control of vibration of a smart CFRP laminated beam, Smart Mater Struct 9 (2000), 215-219.

[29] S.B. Kim, C.B. Yun and B.F. Spencer, Vibration control of wind-excited tall buildings using sliding mode fuzzy control, Journal of Engineering Mechanics-ASCE 130 (2004), 505-510.

[30] W.Z. Qu, J.C. Sun and Y. Qiu, Active control of vibration using a fuzzy control method, Journal of Sound and Vibration 275 (2004), 917-930.

[31] M.D. Claudia, Fuzzy control of magnetorheological dampers for vibration reduction of seismically excited structures, PhD Thesis, Florida State University, 2005

[32] A. Sinha and C.K. Kao, Independent modal sliding mode control of vibration in flexible structure, Journal of Sound and Vibration 147 (1991), 352-358.

[33] F. Wang, Modeling, optimization and vibration control of piezoelectric structures, PhD Thesis, National University of Defense Technology, China, 2006. 

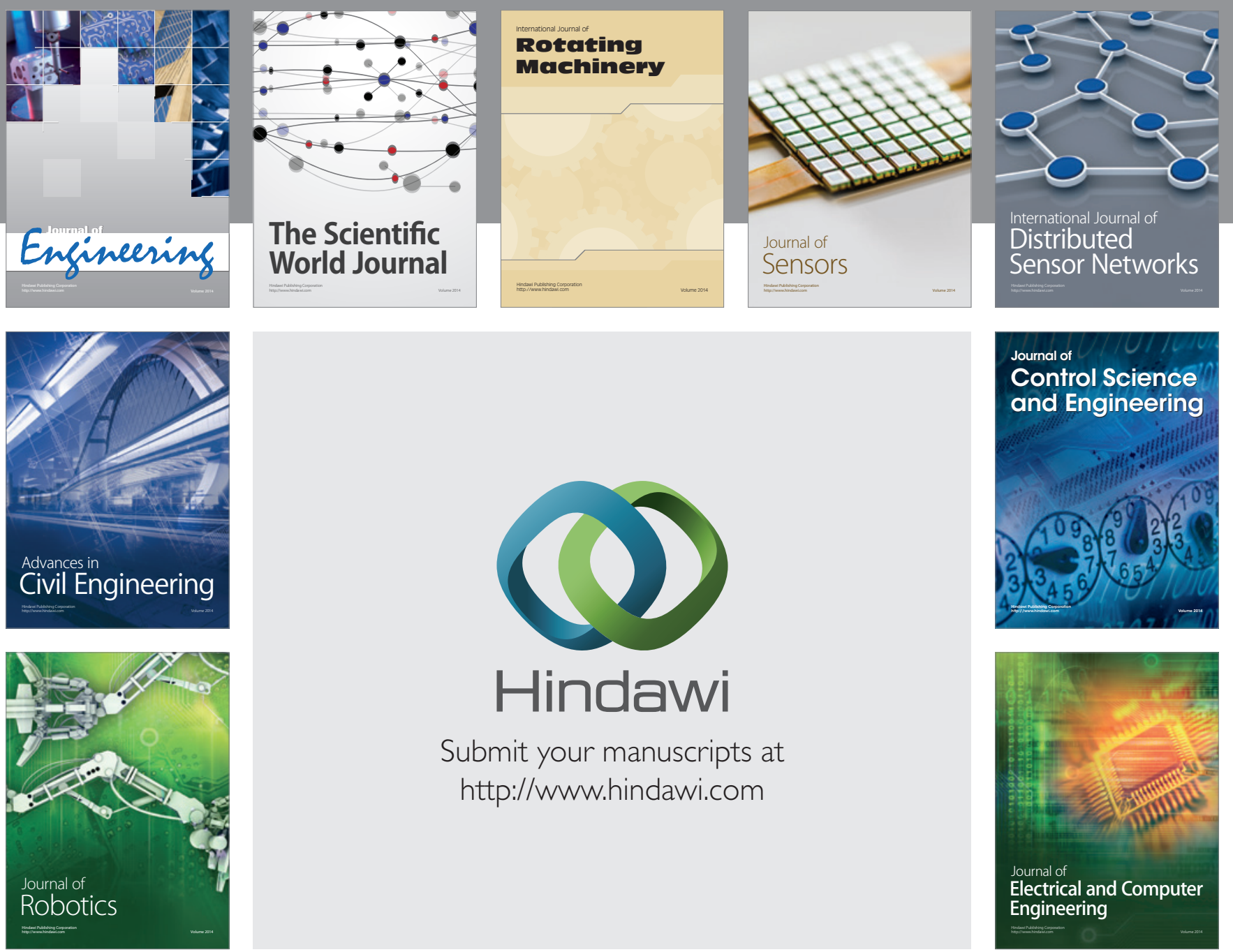

Submit your manuscripts at

http://www.hindawi.com
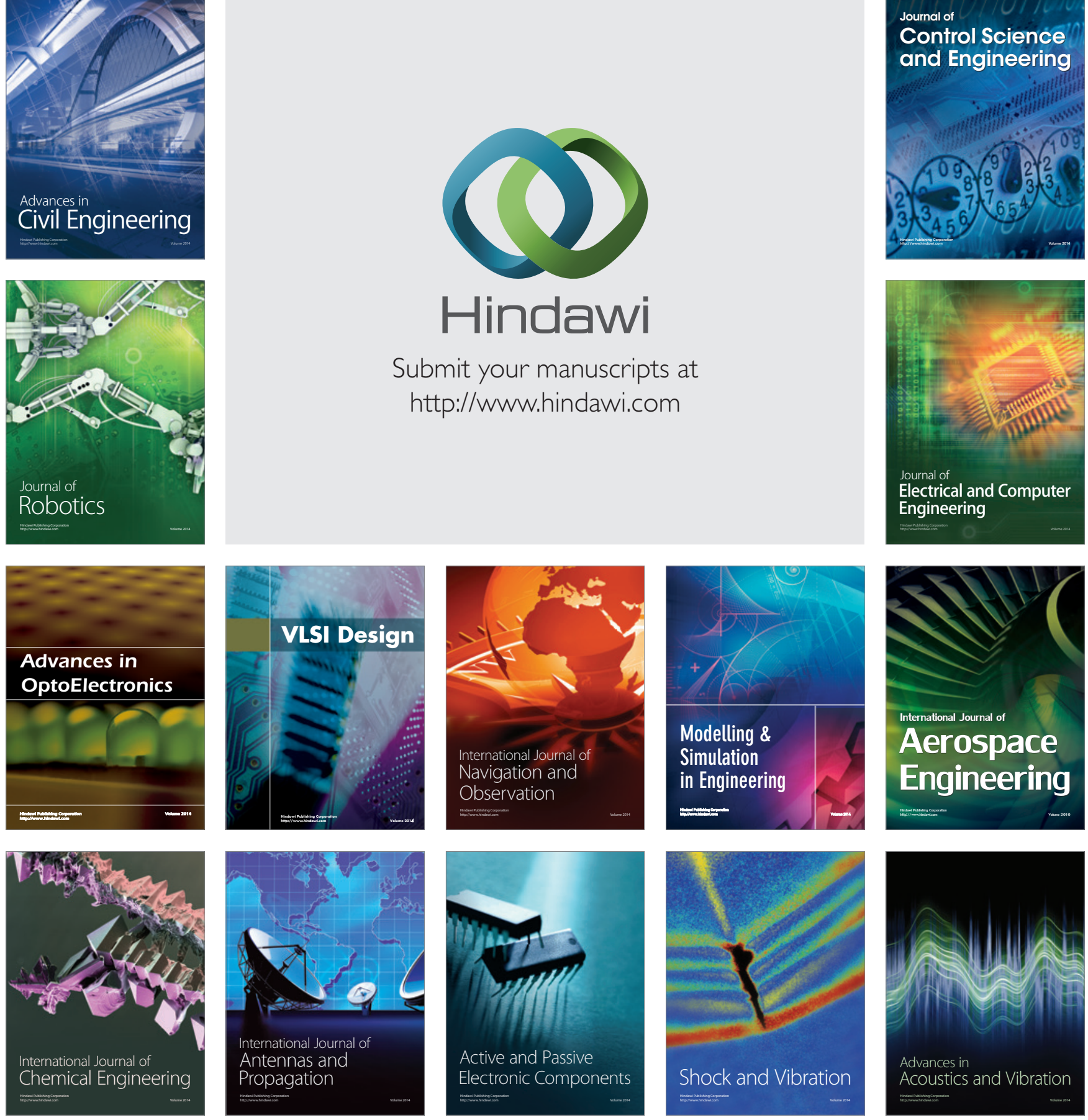